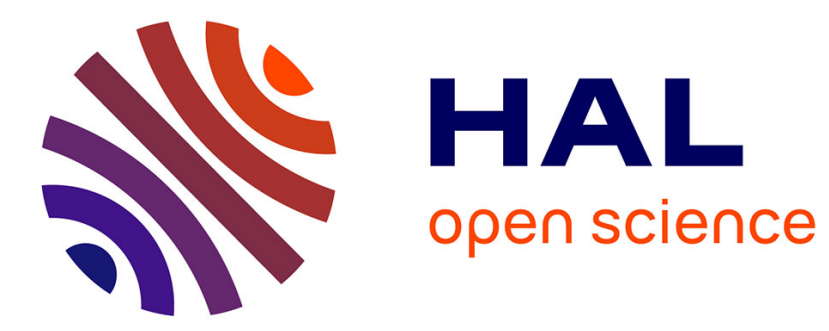

\title{
Microwave Mixing Technique for Nondestructive Measurement of Moisture Content of Particulate Agricultural Products
}

\author{
Chenxiao Li, Yanlei Xu, He Gong, Yuanyuan Liu, Qian Song
}

\section{To cite this version:}

Chenxiao Li, Yanlei Xu, He Gong, Yuanyuan Liu, Qian Song. Microwave Mixing Technique for Nondestructive Measurement of Moisture Content of Particulate Agricultural Products. 11th International Conference on Computer and Computing Technologies in Agriculture (CCTA), Aug 2017, Jilin, China. pp.100-108, 10.1007/978-3-030-06179-1_11 . hal-02111548

\author{
HAL Id: hal-02111548 \\ https://hal.inria.fr/hal-02111548
}

Submitted on 26 Apr 2019

HAL is a multi-disciplinary open access archive for the deposit and dissemination of scientific research documents, whether they are published or not. The documents may come from teaching and research institutions in France or abroad, or from public or private research centers.
L'archive ouverte pluridisciplinaire HAL, est destinée au dépôt et à la diffusion de documents scientifiques de niveau recherche, publiés ou non, émanant des établissements d'enseignement et de recherche français ou étrangers, des laboratoires publics ou privés. 


\title{
Microwave Mixing Technique for Nondestructive
}

\section{Measurement of Moisture Content of Particulate}

\author{
Agricultural Products
}

\author{
Chenxiao Li ${ }^{1, \mathrm{a}}$, Yanlei Xu ${ }^{1, \mathrm{~b}}$, He Gong ${ }^{1, \mathrm{c}}$, Yuanyuan Liu ${ }^{1, \mathrm{~d}}$, and Qian Song ${ }^{2, e,(\bowtie)}$ \\ 1. College of Information, Jilin Agriculture University, Changchun Jilin, China \\ 2. College of Physics, Jilin University, Changchun Jilin, China \\ a. lichenxiao2000@163.com; b. yanleixu@163.com; c. 29878671@qq.com; \\ d. 24973770@qq.com; e. songqian0323@163.com
}

\begin{abstract}
A new method for nondestructive measurement of moisture content of particulate agricultural products is developed using microwave mixing technique. A double horn system with a relative vertical distance of one-fourth wavelengths is designed to measure the reflected microwave signals. The particulate materials are interacted with microwave at a frequency of $10.5 \mathrm{GHz}$, and the reflected microwave signal is mixed with the launch signal. Corns with different moisture contents are chosen as samples. Calibration models for moisture content are proposed in accordance with the measurement of the differential attenuation of the microwave mixing signal. A moisture content from $6.8 \%$ to $30.1 \%$ is obtained with a coefficient of determination of 0.98 and a standard error of calibration value of $1.12 \%$.
\end{abstract}

Keywords: Moisture content; Microwaves; Mixing technique; Nondestructive measuring

\section{Introduction}

Water is an important component of industrial and agricultural production. Moisture content directly affects the quality of storage and processing conditions of agricultural products. The measurement of moisture content has important scientific significance and application value in agriculture product processing applications.

To achieve automatic processing and production, the moisture measurement process of agricultural products needs to be completed accurately and quickly, and the tested samples are not damaged. The drying weighing method is often used as a calibration method. Each measurement requires very few samples.[1,2]. The process is destructive and time and energy consuming. Resistance method needs to measure the impedance of samples between probes, with low accuracy and destructive measurement. RF capacitance method is a non-destructive measurement, in which samples are added between capacitor plates and water content is retrieved by measuring capacitance changes. [3, 4]. However, the method 
results in bulk density of sample, thereby causing a large number of measurement errors. It is an urgent research topic to realize on-line non-destructive and high-precision detection of moisture content in agricultural products.

In recent decades, microwave measurements have been used in many industrial applications. Microwave moisture content sensing measures the relative insensitivity to ionic conductivity, during which the electromagnetic energy is mainly absorbed by water. This selective absorption is due to the polar characteristic of the water molecules and their high permittivity compared with that of dry matter [5,6]. Water molecules have very strong polarity, and microwave has a high frequency, so the interaction between the two will lead to energy storage and loss. The dielectric constant critically depends on the moisture content of water-containing materials at constant frequency and temperature [7]. There are many reports on dielectric constant measurement, including resonant cavity method and transmission line method. [8, 9]. The dielectric constant of the sample is calculated by measuring the stationed Bobbi and field strength of the microwave. During the measurement, the sample is strictly confined to the waveguide cavity or coaxial line, which limits the application of on-line measurement [10].

Free-space technology presents great flexibility and does not bound microwaves to specific regions. After the interaction between microwave and sample, the transmission energy and phase will change. The moisture content and bulk density of sample can be measured by measuring the transmission parameters of microwave [11]. The microwave transmitting and receiving antennas are fixed on both sides of the measured sample. The thickness of the sample should be kept constant during the measurement to determine the relative attenuation of microwave energy. Therefore, the dual probe method cannot be applied to the situation where the thickness of the sample changes continuously during the flow. In our previous studies, a free-space microwave reflection measurement system with a single sensor is designed for the nondestructive determination of moisture content and bulk density [12]. The amplitude and phase parameters of traveling-standing wave are measured through the microwave horn antenna of continuous motion. However, for large particles of material, the single probe measurement sensitivity decreases as microwave scattering increases.

In the present study, a double probe microwave reflection measurement system is designed. The reflected microwave signal is mixed with the launch signal. Two radar probes are fixed separately on the position of signal maxima and minima. Measuring sensitivity is increased by differential output. This method is fast, continuous, and nondestructive. The measuring sensor does not directly contact with the grain materials, thereby facilitating its use in the real-time monitoring and controlling processes of moisture content of industrial and agricultural products. 


\section{Materials and Methods}

The microwave moisture measurement system described here is based on a free-space reflection method and possesses the advantages of being nondestructive and not requiring sample preparation and physical contact with the material. Moisture content is determined from the measurement of attenuation and phase shift after the incident wave reflection from the material surface. A transmit and reflection wave mixing method is designed to determine the parameters of attenuation and phase shift.

\subsection{Principle of Microwave Mixing Moisture Measurement}

The schematic view of this microwaves mixing moisture measurement is shown in Fig. 1. The materials are interacted with microwave, and the reflected microwave signal is mixed with the launch signal in the mixing diode. $U_{L}(t)$ is the incident wave signal with the frequency $\omega_{L}$, and $U_{S}(t)$ is the reflected microwave signal with the frequency $\omega_{S}$. D1 is a Schottky mixing diode. The DC offset voltage $\mathrm{E}_{0}$ is used to make sure the diode working well. Finally, the signals are processed by an amplifier and a low pass filter.

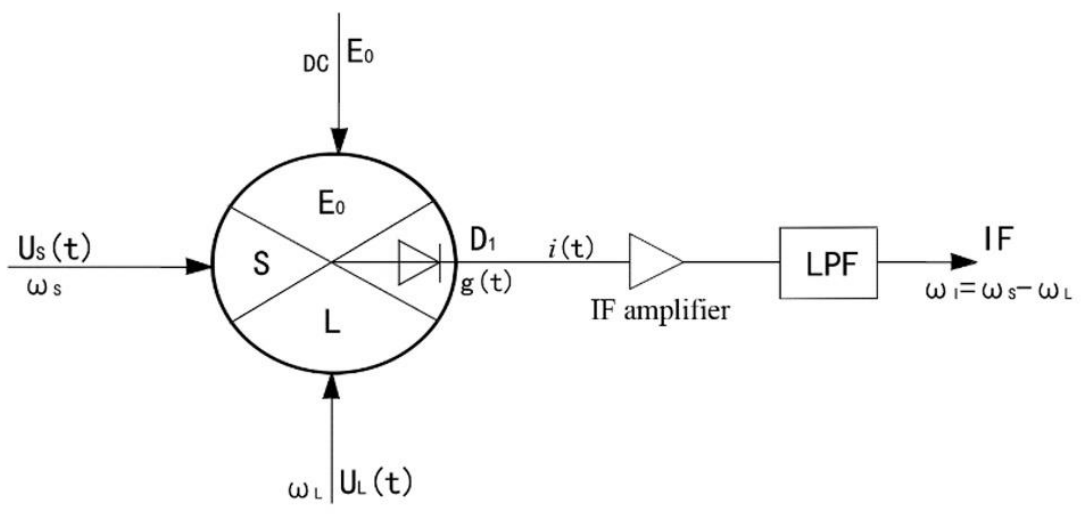

Fig. 1. The schematic view of microwave mixing process

The mixing principle is described as follows. The incident wave signal $U_{L}(t)$ and the reflected microwave signal $U_{S}(t)$ can be described as

$$
\begin{gathered}
\mathrm{U}_{\mathrm{L}}(\mathrm{t})=\mathrm{U}_{\mathrm{L}} \cos \omega_{\mathrm{L}} \mathrm{t}, \\
\mathrm{U}_{\mathrm{S}}(\mathrm{t})=\mathrm{U}_{\mathrm{S}} \cos \left(\omega_{\mathrm{S}} \mathrm{t}+\varphi\right) .
\end{gathered}
$$

The incident and reflected waves have different amplitudes and phases. After contact with the material, the reflected microwave $U_{S}(t)$ is a small variation compared with the incident wave. So the current $\mathrm{i}(\mathrm{t})$ flowing through the Schottky diode mixer $\mathrm{D}_{1}$ can be expanded as Taylor series

$$
\mathrm{i}(\mathrm{t})=\mathrm{f}\left(\mathrm{E}_{0}+\mathrm{U}_{\mathrm{L}}(\mathrm{t})+\mathrm{U}_{\mathrm{S}}(\mathrm{t})\right)
$$




$$
\begin{aligned}
=f\left(E_{0}+\right. & \left.U_{L} \cos \omega_{L} t\right)+f^{\prime}\left(E_{0}+U_{L} \cos \omega_{L} t\right) U_{S} \cos \left(\omega_{S} t+\varphi\right) \\
& +\frac{1}{2 !} f^{\prime \prime}\left(E_{0}+U_{L} \cos \omega_{L} t\right)\left(U_{S} \cos \left(\omega_{S} t+\varphi\right)\right)^{2}+\Lambda .
\end{aligned}
$$

The first expansion is a large signal, which contains the DC and local microwave fundamental signals. Other items are small signals. For the second item of Eqs. (3)

$$
\begin{aligned}
i(t)=f^{\prime}\left(E_{0}+U_{L} \cos \omega_{L} t\right) U_{S} \cos \left(\omega_{S} t+\varphi\right) \\
=g_{0} U_{S} \cos \left(\omega_{S} t+\varphi\right)+\sum_{n=1}^{\infty} g_{n} U_{S} \cos \left(\left(n \omega_{L}+\omega_{S}\right) t+\varphi\right) \\
+\sum_{n=1}^{\infty} g_{n} U_{S} \cos \left(\left(n \omega_{L}-\omega_{S}\right) t+\varphi\right)
\end{aligned}
$$

In (4), $g_{n}$ is the conductance of the diode mixer and it is a constant for the given diode. After the mixing signals passing through the low-pass filter, the differential frequency signal, at the point $\mathrm{n}=1$, can be expressed as follows

$$
i(t)=g_{1} U_{S} \cos \left(\left(\omega_{L}-\omega_{S}\right) t+\varphi\right) .
$$

In actual measurement, the relative distance between the microwave probe and the material remains constant. So the frequency of the incident wave is equivalent to the reflected microwave. Then we can get the following equation

$$
\mathrm{I}_{0}=\mathrm{g}_{1} \mathrm{U}_{\mathrm{s}} \cos \varphi
$$

In (6), $\mathrm{g}_{1}$ is the o conductance constant of the diode. $\mathrm{U}_{\mathrm{S}}$ is the amplitude of reflected microwave, which is proportional to the moisture content of tested materials. $\varphi$ is the relative phase value of the reflection microwave. The value $\cos \varphi$ varies periodically from 1 to -1 with the different relative position of the antenna probe and the material. The mixing signal has the best sensitivity when the microwave antenna is fixed in one of the two phase extreme points $(\cos \varphi=1$ or -1$)$.

In this study, two microwave antennas are mounted on the maximum and minimum phase positions respectively. In experimental measurement, two mixing signals are opposite to each other. The moisture content of the material is obtained by difference operation of the two signals. Microwave mixing technique has greatly facilitated the high-frequency signal processing and extraction in moisture measurement.

\subsection{Microwave Measurement System}

The measurement system consists of microwave cavity oscillator, double horn antenna, mixer, sample holder, differential amplifier, and data processing unit. The construction of microwave measurement system is illustrated in Fig. 2. Two microwave horn antennas, which transmit and receive signals independently, are mounted on the same side of the sample holder. The sample container is made of acrylic material, rectangular and filled with 
the tested sample. The vertical distance of microwave transceiver probes 1 and 2 is $\lambda / 4$, which relates to microwave wavelengths. Prior to the actual measurement, the distance $\mathrm{L}$ between probe 2 and the sample surface needs to be adjusted to ensure that the mixing signal is at the limit value. When $\mathrm{L}$ is finalized, the phase difference $\varphi$ between mixing signals $\mathrm{A}$ and $\mathrm{B}$ is $\pi$. Therefore, the mixing current presents opposite changes between signals $\mathrm{A}$ and $\mathrm{B}$, as shown in Eq. (6).

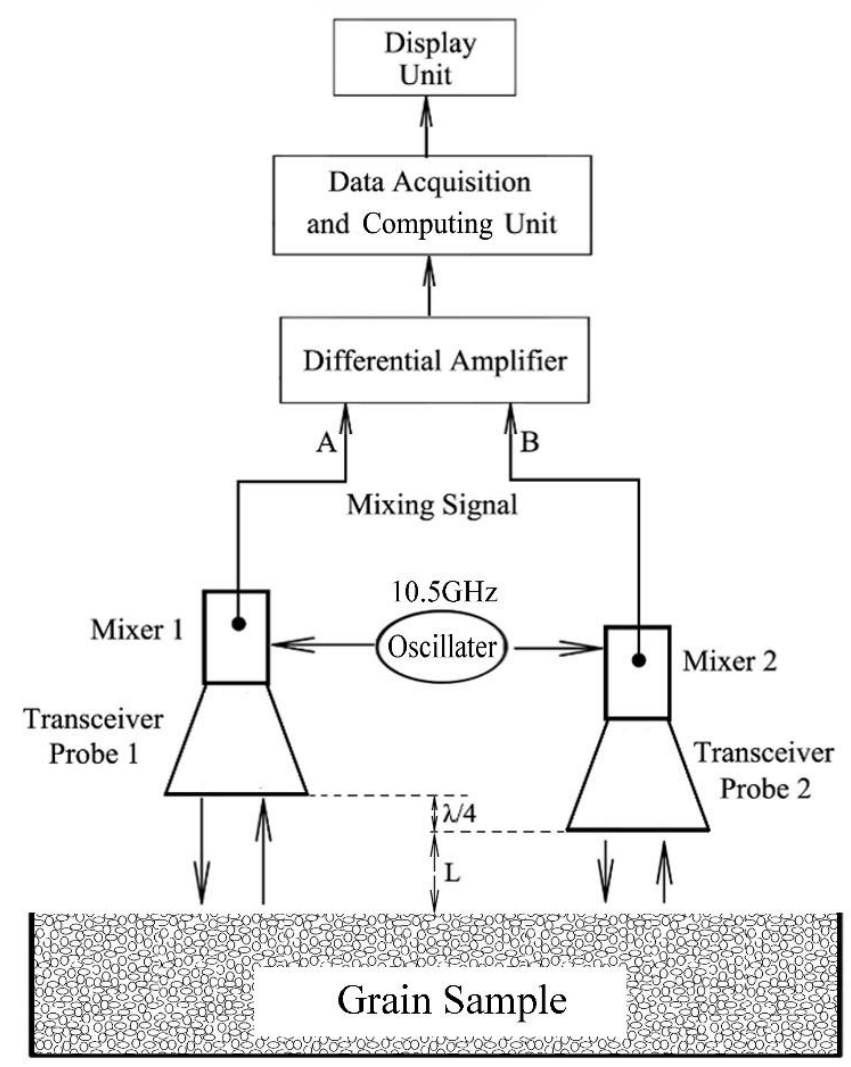

Fig. 2. Schematic diagram of double horn antenna microwave measurement system

The microwave sensors are designed as follows. The continuous microwave signal with a frequency of $10.5 \mathrm{GHz}$ and a power of $20 \mathrm{~mW}$ is generated by a Gunn diode. The waveguide cavity is wr90, and the oscillating diode is mounted in the waveguide cavity. The microwave cavity oscillator is connected with the horn antenna. The aperture of the microwave horn antenna is $8.4 \mathrm{~cm} \times 6 \mathrm{~cm}$ and the output gain at the frequency of $10.5 \mathrm{GHz}$ is $17.2 \mathrm{dBi}$. The incident and reflected waves are detected by a Schottky mixer diode. Finally, mixing signals $\mathrm{A}$ and $\mathrm{B}$ are measured by using the differential amplifier.

The output voltage of the differential amplifier is linearly related to the moisture content of the tested materials. In accordance with the fitting equation, a date acquisition and 
adjustment unit is designed with different modes to realize the display and control of moisture content.

\subsection{Sample Preparation}

Corns are used as the experimental sample in this study. Each sample consists of approximately $10 \mathrm{~kg}$ of material and is dried by natural drying prior to the experiments. The moisture content of the sample is determined by drying and weighing. A certain amount of the sample is placed in the drying equipment at a temperature of $130^{\circ} \mathrm{C}$ until the weight is constant. Moisture content is calculated as follows:

$$
\mathrm{M}(\%)=\mathrm{m}_{\mathrm{w}} /\left(\mathrm{m}_{\mathrm{w}}+\mathrm{m}_{\mathrm{d}}\right),
$$

where $\mathrm{M}$ is the moisture content of corn, $\mathrm{m}_{\mathrm{w}}$ is the mass of water, and $\mathrm{m}_{\mathrm{d}}$ is the mass of dry matter.

The initial moisture content of corn sample is $6.8 \%$. The water content of the sample was increased by adding water and stirring evenly. The samples were prepared with the distance of 3\% water content. A total of 8 samples with moisture contents from $6.8 \%$ to $30.1 \%$ for corn are prepared. Samples are placed in a sealed plastic bag for at least $24 \mathrm{~h}$ at $4{ }^{\circ} \mathrm{C}$ to equilibrate. Before the measurement, the samples were transferred to room temperature for at least 24 hours to achieve a temperature balance.

\subsection{Measurement Procedure}

The sample container is filled with corn samples and placed on the side of the microwave antenna. The surface of the sample is perpendicular to the direction of microwave radiation. The size of the sample container should be larger than the microwave antenna to reduce the edge scattering interference of the microwave.

The microwave horn antenna is fixed on the sliding orbit, and the position of the antenna can be adjusted freely. The distance between antenna probe 2 and sample surface is L1. In measurement, the electric field of space microwave varies periodically with the change of L1. The voltage signal B detected by the digital voltmeter changes with the increase in horn antenna distance. The variations in voltage signal $\mathrm{B}$ with the antenna distance $\mathrm{L}$ for corn with an initial moisture content of 6.8\% are illustrated in Fig. 3. 


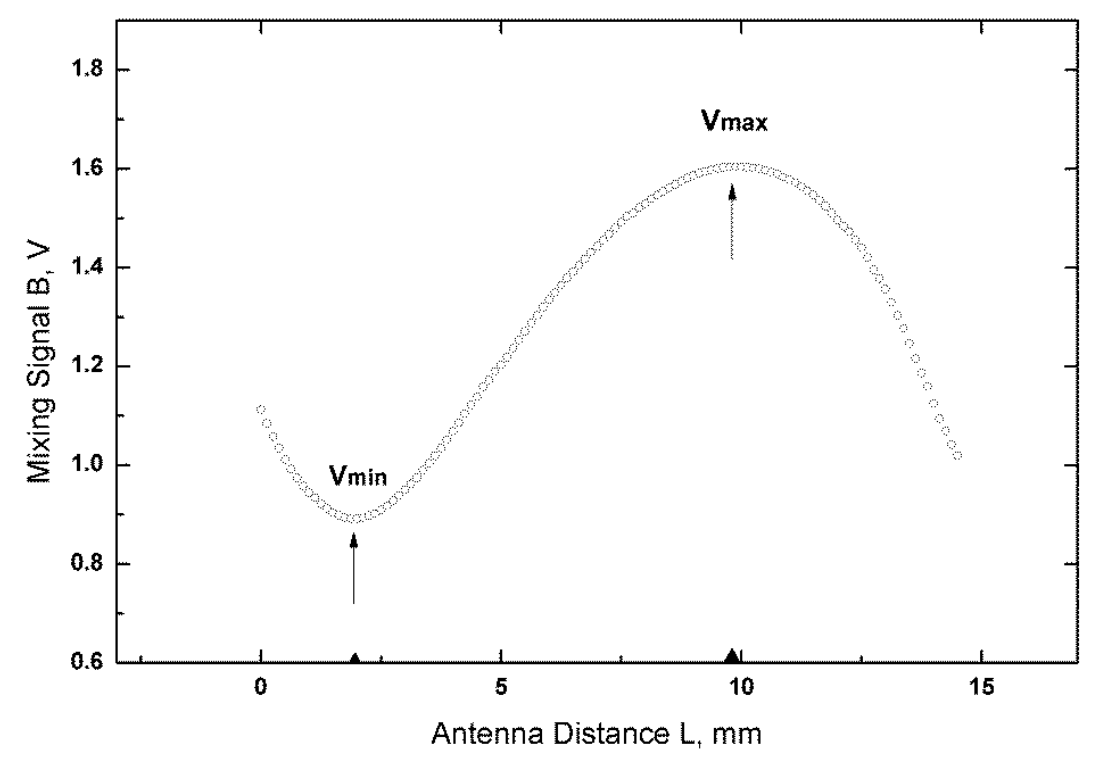

Fig. 3. Variation of voltage signal $B$ with antenna distance $\mathrm{L}$ at the moisture content of $6.8 \%$

Two extreme points, Vmin and Vmax, appear with the increase in horn antenna distance. The corresponding antenna distances are 2.4 and $9.8 \mathrm{~mm}$. During the experiment, two microwave antenna probes are fixed in the two positions. Extreme position distance is 7.4 $\mathrm{mm}$, which is in good agreement with the theoretical calculations of $\lambda / 4$ at $10.5 \mathrm{GHz}$.

For each corn sample with specific moisture content, measurements are repeated and performed three times. Mixing voltage signals A and B are recorded by using the digital voltmeter, and the differential values are calculated for data fitting.

\section{Experimental Results}

The relationship between moisture contents and mixing voltage signals of corn samples is shown in Fig. 4. The sample is measured at room temperature $\left(24{ }^{\circ} \mathrm{C}\right)$ with eight different moisture contents in increments of approximately 3\%. The moisture contents vary from $6.8 \%$ to $30.1 \%$. Each of the samples is repeated for three times, and 48 sets of data are recorded.

Curves A and B represent the output of the two microwave probes. The trends of A and B variation with moisture content are opposite to each other because the phase difference between the mixing signals is $\pi$. Curve B presents a greater slope than curve A because of the close measuring distance. 


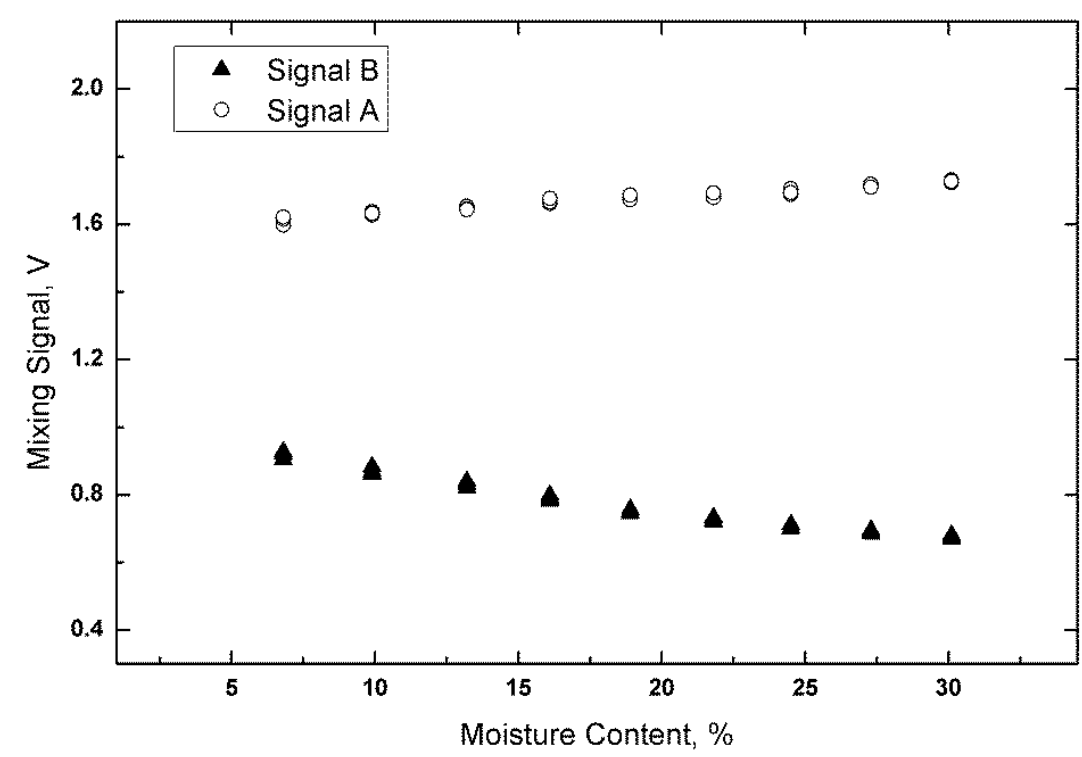

Fig. 4. The variations in the mixing signal with moisture content. The round and triangle data are output of probe 1 and probe 2 respectively.

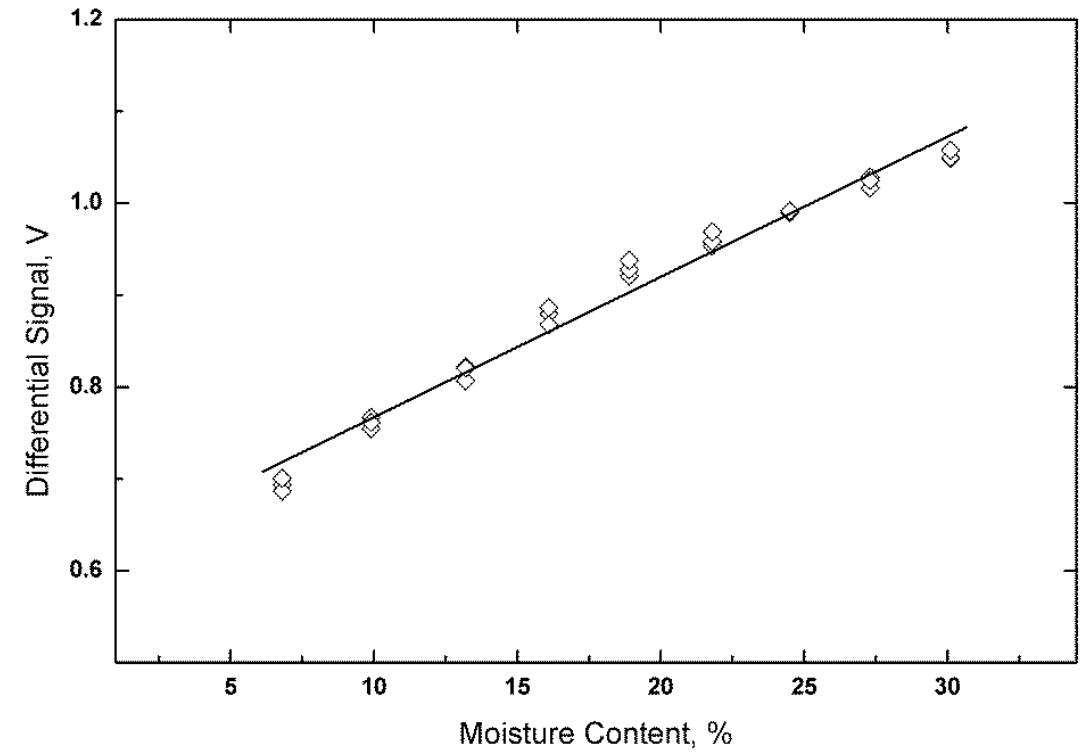

Fig. 5. Differential mixing signal versus the moisture content of corn

The variations in the differential mixing signals with moisture content are illustrated in Fig. 5. The sensitivity of the differential signal is obviously improved compared with that of 
a single mixing output probe 1 or probe 2 . The differential output is generally linear. Therefore, calibration equation is assumed as follows:

$$
\mathrm{M}_{\mathrm{C}}(\%)=\mathrm{a}_{0} \mathrm{U}_{\mathrm{d}}+\mathrm{b}_{0},
$$

where $a_{0}$ and $b_{0}$ are the coefficients, $U_{d}$ is the differential signal voltage, and $M_{C}$ is the moisture content of corn. The calibration equation is obtained by statistically analyzing all experimental data to determine the coefficients. The results show that, the coefficients $\mathrm{a}_{0}$ and $\mathrm{b}_{0}$ are 63.86 and 38.79, respectively. The moisture content is determined with an $\mathrm{R}^{2}$ of 0.98 , and the standard error of calibration (SEC) is $1.12 \%$.

Particulate material microwave scattering is a major error factor. The main source of microwave scattering is the inhomogeneity of sample distribution, which is affected by the size, shape and water content of the particles. Temperature and bulk density also affect the measurement results. In the experiment, the particle of materials should be stacked compactly and orderly to reduce the irregular scattering of microwaves.

\section{Conclusion}

A microwave mixing system is designed for the rapid and nondestructive determination of moisture content of gain materials. The measurement system consists of microwave cavity oscillator, double horn antenna, mixer, sample holder, differential amplifier, and data processing unit. The reflected microwave signal is mixed with the launch signal. Measuring sensitivity is increased by using the double probe microwave reflection measurement system. In accordance with the fitting calibration equation, the moisture content of corn is determined with a coefficient of determination $\left(\mathrm{R}^{2}\right)$ of 0.98 and a standard SEC of $1.12 \%$. The proposed measuring method is suitable for the independent moisture measurement of monomers and on-line moisture measurement, and also meets the real-time monitoring needs of agricultural products moisture change in industry.

Acknowledgment. This work was supported by Jilin Scientific and Technological Development Program (No. 20180520016JH) and the Scientific Research Foundation of Jilin Agricultural University (No. 201719).

\section{References}

[1] E. Martines-López, L. Lira-Cortés. Measurement of Moisture Content of Some Hydrated Ionic Materials by Oven Drying[J]. Mapan, 2015, 30(4):239-247.

[2] Walker J P, Willgoose G R, Kalma J D. In situ measurement of soil moisture: a comparison of techniques | NOVA. The University of Newcastle's Digital Repository[J]. Journal of Hydrology, 2004, 293(1-4):85-99.

[3] Wu K. Measurement of soil moisture change in spatially heterogeneous weathered soils using a 
capacitance probe [J]. Hydrological Processes, 2015, 12(1):135-146.

[4] Kim K B, Kim J H, Lee S S, et al. Measurement of grain moisture content using microwave attenuation at $10.5 \mathrm{GHz}$ and moisture density[J]. Instrumentation \& Measurement IEEE Transactions on, 2000, 51(1):72-77.

[5] Nelson S O, Trabelsi S. Factors influencing the dielectric properties of agricultural and food products.[J]. Journal of Microwave Power \& Electromagnetic Energy A Publication of the International Microwave Power Institute, 2012, 46(2):93-107.

[6] Trabelsi S, Nelson S O. Nondestructive sensing of physical properties of granular materials by microwave permittivity measurement[J]. IEEE Transactions on Instrumentation \& Measurement, 2006, 55(3):953-963.

[7] Trabelsi S, Lewis M A, Nelson S O. Microwave moisture meter for in-shell peanut kernels[J]. Food Control, 2016, 66:283-290.

[8] Krupka J. REVIEW ARTICLE:, Frequency domain complex permittivity measurements at microwave frequencies[J]. Measurement Science \& Technology, 2006, 17(6):55-70.

[9] Hashemi A, Rashidi M, Kurtis K E, et al. Microwave dielectric properties measurements of sodium and potassium water glasses[J]. Materials Letters, 2016, 169:10-12.

[10] Nigmatullin R, Nelson S O. New quantitative "reading" of dielectric spectra of complex biological systems[J]. Dielectrics \& Electrical Insulation IEEE Transactions on, 2006, 13(6):1325-1334.

[11] Trabelsi S, Paz A M, Nelson S O. Microwave dielectric method for the rapid, non-destructive determination of bulk density and moisture content of peanut hull pellets[J]. Biosystems Engineering, 2013, 115(3):332-338.

[12] Li C, Han B, Zhang T. Free-space reflection method for measuring moisture content and bulk density of particulate materials at microwave frequency.[J]. Review of Scientific Instruments, 2015, 86(3):227-237 